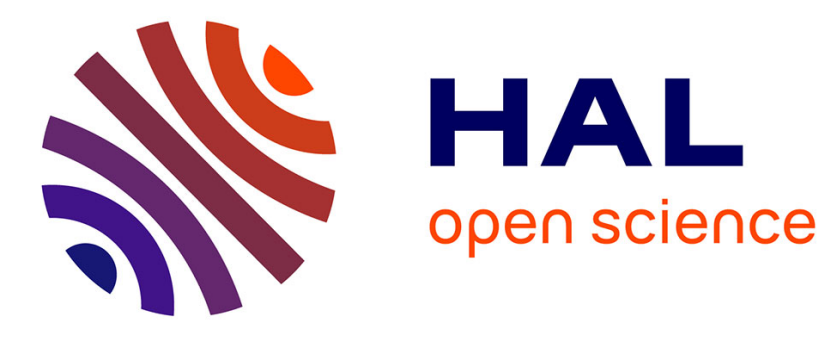

\title{
Modélisation dynamique du thermoformage
}

Erwan Verron, Gilles Marckmann, Bernard Peseux

\section{To cite this version:}

Erwan Verron, Gilles Marckmann, Bernard Peseux. Modélisation dynamique du thermoformage. Revue Européenne des Éléments Finis, 1998, 7 (1 2 3), pp.193-208. 10.1080/12506559.1998.11690474 . hal-01007073

\section{HAL Id: hal-01007073 https://hal.science/hal-01007073}

Submitted on 8 Oct 2016

HAL is a multi-disciplinary open access archive for the deposit and dissemination of scientific research documents, whether they are published or not. The documents may come from teaching and research institutions in France or abroad, or from public or private research centers.
L'archive ouverte pluridisciplinaire HAL, est destinée au dépôt et à la diffusion de documents scientifiques de niveau recherche, publiés ou non, émanant des établissements d'enseignement et de recherche français ou étrangers, des laboratoires publics ou privés. 


\title{
Modélisation dynamique du thermoformage
}

\section{Erwan Verron - Gilles Marckmann - Bernard Peseux}

\author{
Laboratoire Mécanique et Matériaux, Division Structures \\ Ecole Centrale de Nantes \\ BP 92101, 44321 Nantes cedex 3
}

RÉSUMÉ. Un code de calcul par éléments finis a ểé développé pour simuler le procédé industriel de thermoformage. Le modèle numérique repose sur une formulation lagrangienne totale en dynamique et l'équation du mouvement est intégrée par un schéma explicite. Les feuilles de polymère chauffées sont modélisées par des membranes incompressibles hyperélastiques ou viscoélastiques non linaires. Le contact est supposé collant et un module de raffinement de maillage a été implanté. Les résultats fournis par le code de calcul sont comparés à des résultats semi-analytiques et expérimentaux et un exemple complexe montre les bonnes performances du modele.

ABSTRACT. A numerical sofiware was developed ta simulate the thermoforming process. The numerical model is based on a total Lagrangian finite element method and the equation of motion is integrated via an explicit scheme. The heat-softened polymer sheets are modeled by hyperelastic or non-linear viscoelastic incompressible membranes, the usual assomption of sticking contact is adopted and an adaptative mesh refinement scheme was implemented. The finite element results are compared with semi-analytical and exprimental results and successful calculations are made using a critical mold geometry.

MoTs-CLÉs : thermoformage, éléments finis, membrane, hyperélasticité, viscoélasticité non linaire, schéma dynamique explicite, raffinement de maillage.

KEY WORDS: thermoforming, finite elements method, membrane, hyperelasticily, nonlinear viscoelasticity, dynamic explicit scheme, mesh refinement.

\section{Introduction}

Les matières plastiques occupent actuellement une place grandissante dans de nombreux secteurs de l'industrie tels que l'automobile, l'isolation en électronique et, bien sûr, l'cmballage. Dans tous ces domaines, la mise en forme de ces matériaux est un problème d'actualité qui fait l'objet de nombreuses recherches. La mise en forme des matières plastiques est devenue un secteur d'activité à part entière. 
Parmi les différents procédés industriels de mise en forme, le thermoformage permet la fabrication de corps creux ouverts (figure 1).

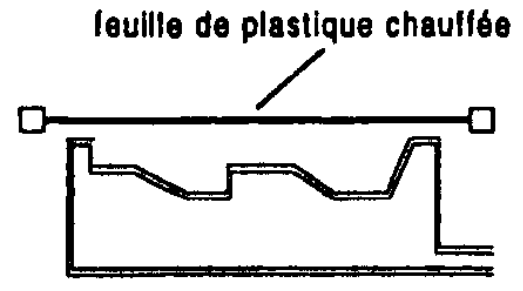

(1)

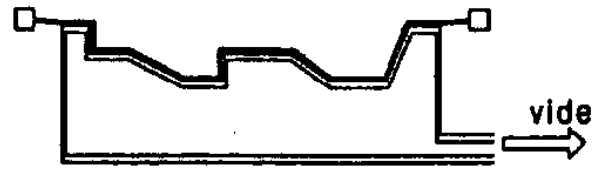

(2)

Figure 1. Différentes étapes du procédé de thermoformage

Dans ce cadre, l'objectif de la simulation numérique est de fournir des outils d'aide à la conception des moules et de permettre une meilleure maîtrise du procédé tout en optimisant la quantité de matière utilisée. Les programmes actuels de simulation utilisent, le plus souvent, la méthode des éléments finis et des lois de comportement hyperelastiques dans un cadre quasi-statique [ZAM 89], [DEL 91].

Cependant, les très fortes non-linéarités rencontrées, aussi bien matérielles que géométriques, mais aussi et surtout l'apparition de phénomènes d'instabilité lors du gonflement [KHA 92] engendrent des problèmes de convergence. Pour remédier à ces difficultés, nous avons développé un code de calcul utilisant un schéma de résolution dynamique explicite, habituellement rencontré dans la simulation de l'emboutissage et récemment étendu au cas de la mise en forme des plastiques par Rachik et al. [RAC 94]. Ce logiciel intègre des lois de comportement aussi bien hyperélastiques que viscoélastiques non-linéaires ainsi qu'un module de remaillage permettant de s'adapter à l'évolution de la géométric.

\section{Mise en équations}

\subsection{Formulation variationnelle}

Dans les problèmes de soufflage, la paraison initiale est supposée entièrement connue et définie. Pour cette raison, nous nous sommes tournés vers une formulation lagrangienne totale du problème : la configuration de référence reste la configuration initiale tout au long du calcul. En l'absence de forces de volume, le Principe des Travaux Virtuels s'écrit :

$$
\begin{aligned}
\iiint_{V_{0}} \delta \vec{u} \cdot \rho_{0} \ddot{\vec{u}}(\vec{X}, \tau) d V_{0}=-\iiint_{V_{0}} \delta \overline{\bar{E}}: \overline{\bar{S}} d V_{0}+\iint_{S_{0} \tau} \delta \vec{u} \cdot \overrightarrow{T_{0}} d S_{0} \\
\forall \delta \vec{u}^{t} \text { compatible }
\end{aligned}
$$

où $V_{0}$ et $S_{0 T}$ sont respectivement le volume et la surface frontière sur laquelle le chargement est imposé dans l'état initial non déformé, $\rho_{0}$ est la masse volumique, 
$\ddot{\vec{u}}(\vec{X}, \tau)$ le vecteur accélération exprimé en fonction de la position initiale, $\overline{\bar{E}}$ le tenseur des déformations de Green-Lagrange, $\overline{\bar{S}}$ le second tenseur des contraintes de Piola-Khirchhoff, $\overrightarrow{T_{0}}$ la densité surfacique de force relativement à la configuration initiale et $\delta \vec{u}$ un déplacement virtuel compatible.

\subsection{Discrétisation spatiale : éléments finis}

Le domaine est discrétisé en éléments finis et le système discret à résoudre est le suivant :

$$
\{\mathcal{A}\}=-\left\{\mathcal{F}_{\text {int }}\right\}+\left\{\mathcal{F}_{\text {ext }}\right\}
$$

où les trois vecteurs sont respectivement les vecteurs des quantités d'accélération, des forces intérieures et des forces extérieures donnés par :

$$
\begin{gathered}
\{\mathcal{A}\}=\sum_{i=1}^{n_{e}} \iiint_{V_{0_{i}}}[N]^{t} \rho_{0}[N]\{\ddot{U}\} d V_{0} \\
\left\{\mathcal{F}_{\text {int }}\right\}=\sum_{i=1}^{n_{e}} \iiint_{V_{\delta_{i}}}[B]^{t}\left\{S^{e}\right\} d V_{0} \\
\left\{\mathcal{F}_{\text {ext }}\right\}=\sum_{i=1}^{n_{e}} \iint_{S_{\text {o }}^{e}}[N]^{t}\left\{T_{0}^{e}\right\} d S_{0}
\end{gathered}
$$

avec $n_{e}$ le nombre d'éléments finis, $V_{0_{i}}^{e}$ et $S_{0 T_{i}}^{e}$ le volume et la surface (où le chargement est imposé) élémentaires, $[N]$ la matrice d'interpolation, $\{\ddot{U}\}$ le vecteur nodal élémentaire des accélérations, $[B]$ la matrice liant les déformations élémentaires au vecteur des déplacements nodaux élémentaires, $\left\{S^{e}\right\}$ le vecteur des contraintes de Piola-Khirchhoff de seconde espèce et $\left\{T_{0}^{e}\right\}$ le vecteur des forces de surface.

Dans ce travail, on utilise, comme la plupart des auteurs [ZAM 89], des éléments membranes triangulaires à trois nœuds et trois degrés de liberté par nœuds. Ces éléments présentent l'avantage d'une intégration spatiale exacte et rapide. En effet, compte tenu de la linéarité de l'interpolation, les états de déformation et de contrainte sont constants dans l'élément.

De plus, l'hypothèse d'un comportement de type membrane impose un état de contrainte plan dans le plan de l'élément déformé. On décompose la transformation d'un élément en un mouvement de solide rigide puis une déformation locale membranaire dans ce plan (figure 2).

En notant $A, B, C$ les trois noeuds du triangle non déformé, $(X, Y)$ les coordonnées de ces points dans le repère local et $(u, v)$ leurs déplacements, la matrice $[B]$, permettant de relier le vecteur local des déformations de Green-Lagrange 


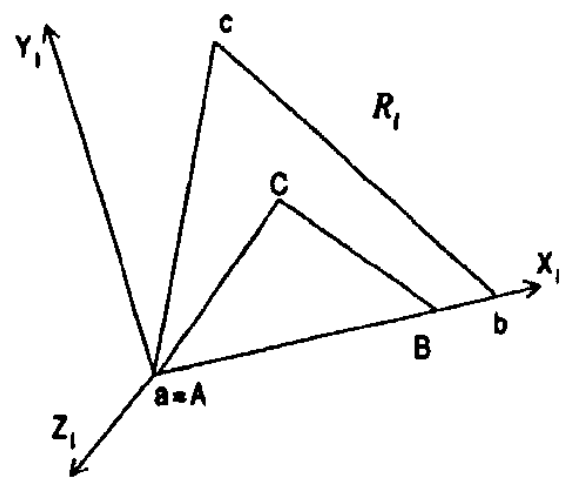

Figure 2. Déformation d'un élément fini membrane dans son plan : $(A B C)$ élément non déformé et $(a b c)$ élément déformé

$\left\{E_{l}\right\}^{t}=\left\langle E_{11}, E_{22}, E_{12}\right\rangle$ au vecteur des inconnues nodales, s'écrit comme la somme d'une matrice relative à la composante linéaire des déformations, $\left[B^{L}\right]$, et d'une matrice relative à la composante non-linéaire, $\left[B^{N L}\right]$, définies par :

$$
\left[B^{L}\right]^{t}=\left[\begin{array}{ccc}
-\frac{1}{X_{B}} & 0 & \frac{1}{Y_{C}}\left(\frac{X_{C}}{X_{B}}-1\right) \\
0 & \frac{1}{Y_{C}}\left(\frac{X_{C}}{X_{B}}-1\right) & -\frac{1}{X_{B}} \\
\frac{1}{X_{B}} & 0 & -\frac{1}{Y_{C}} \frac{X_{C}}{X_{B}} \\
0 & -\frac{1}{Y_{C}} \frac{X_{C}}{X_{B}} & \frac{1}{X_{B}} \\
0 & 0 & \frac{1}{Y_{C}} \\
0 & \frac{1}{Y_{C}} & 0
\end{array}\right]
$$

et :

$$
\begin{aligned}
& {\left[B^{N L}\right]^{\ell}=} \\
& {\left[\begin{array}{ccc}
-\frac{u_{B}}{X_{B}^{2}} & \frac{1}{Y_{C}^{2}}\left(\frac{X_{C}}{X_{B}}-1\right)\left(u_{C}-\frac{X_{C} u_{B}}{X_{B}}\right) & \frac{1}{X_{B} Y_{C}}\left(\frac{X_{C}}{X_{B}} u_{B}-\frac{u_{B}+u_{C}}{2}\right) \\
0 & \frac{1}{Y_{C}^{2}}\left(\frac{X_{C}}{X_{B}}-1\right) v_{C} & -\frac{v_{C}}{2 X_{B} Y_{C}} \\
\frac{u_{B}}{X_{B}^{2}} & \frac{X_{C}}{X_{B} Y_{C}^{2}}\left(u_{C}-\frac{X_{C} u_{B}}{X_{B}}\right) & \frac{1}{X_{B} Y_{C}}\left(-\frac{X_{C}}{X_{B}} u_{B}+\frac{u_{C}}{2}\right) \\
0 & -\frac{X_{C}}{X_{B} Y_{C}^{2}} v_{C} & \frac{v_{C}}{2 X_{B} Y_{C}} \\
0 & \frac{1}{Y_{C}^{2}}\left(u_{C}-\frac{X_{C} u_{B}}{X_{B}}\right) & \frac{v_{C}}{Y_{C}^{2}} \\
0 & \frac{v_{C}}{Y_{C}^{2}} & 0
\end{array}\right]}
\end{aligned}
$$


Les vecteurs élémentaires des forces intéricures, extéricures et des quantités d'accélération, exprimés dans le repère local sont respectivement :

$$
\begin{gathered}
\left\{F_{\text {int }}^{e}\right\}_{l}=H_{0} S_{0}[B]^{t}\left\{S_{l}\right\} \\
\left\{F_{\text {ext }}^{e}\right\}_{l}=P S\left\langle\begin{array}{llllllllll}
0 & 0 & \frac{1}{3} & 0 & 0 & \frac{1}{3} & 0 & 0 & \frac{1}{3}
\end{array}\right\rangle^{t} \\
\left\{A^{e}\right\}_{l}=\left[M^{e}\right]\{\ddot{U}\}
\end{gathered}
$$

où $H_{0}$ et $S_{0}$ sont l'épaisscur et la surface initiale de l'élément, $\left\{S_{l}\right\}$ le vecteur des contraintes non nulles dans le repère local, $P$ la pression exercée sur l'élément (chargement), $S$ la surface de l'élément déformé et $\left[M^{e}\right]$ la matrice masse élémentaire donnée par :

$$
\left[M^{e}\right]=\frac{1}{12} \rho_{0} S_{0} H_{0}\left[\begin{array}{ccc}
2 I & I & I \\
\cdot & 2 I & I \\
\mathrm{sym} & \cdot & 2 I
\end{array}\right]
$$

\subsection{Discrétisation temporelle}

Une fois assemblés les vecteurs et les matrices élémentaires calculés dans le paragraphe précédent, le temps est discrétisé et le problème global à résoudre au temps $t_{n}$ devient :

$$
[M]\left\{\ddot{U}\left(t_{n}\right)\right\}=\left\{F_{\text {ext }}\left(t_{n}\right)\right\}-\left\{F_{i n t}\left(t_{n}\right)\right\}
$$

On utilise le schéma des différences centrées [DOK 89], conditionnellement stable :

$$
\dot{u}\left(t_{n}\right)=\frac{u\left(t_{n+1}\right)-u\left(t_{n-1}\right)}{2 \Delta t} \quad \text { et } \quad \ddot{u}\left(t_{n}\right)=\frac{u\left(t_{n+1}\right)-2 u\left(t_{n}\right)+u\left(t_{n-1}\right)}{\Delta t^{2}}
$$

Le problème [12] se met alors sous la forme suivante :

$$
\frac{[M]}{\Delta t^{2}}\left\{U\left(t_{n+1}\right)\right\}=\left\{F_{\text {ext }}\left(t_{n}\right)\right\}-\left\{F_{\text {int }}\left(t_{n}\right)\right\}+\frac{[M]}{\Delta t^{2}}\left(2\left\{U\left(t_{n}\right)\right\}-\left\{U\left(t_{n-1}\right)\right\}\right)
$$

Classiquement, dans les problèmes de mise en forme, les déplacements initiaux et vitesses initiales sont nuls pour tous les næuds.

\subsection{Diagonalisation de la matrice masse}

Pour éviter le calcul de l'inverse de la matrice masse et pour n'avoir à effectuer que des calculs vectoriels, on élimine le couplage entre les degrés de liberté en concentrant 
la masse des éléments aux nœuds. Le nombre d'opérations est ainsi considórablement réduit.

Pour ce faire, on utilise la méthode dite «Special Lumping Technique» [ZIE 94], méthode particulièrement efficace pour les problèmes de mécanique des solides et qui fournit les taux de convergence optimaux. On calcule la masse totale élémentaire en sommant tous les termes de la matrice masse d'un élément, $\left[M^{e}\right][11]$, et l'on redistribue cette somme proportionnellement aux termes de la diagonale. Dans notre cas, la matrice masse élémentaire diagonale obtenue est :

$$
\left[\overline{M^{e}}\right]=\frac{\rho_{0} S_{0} H_{0}}{3}[I]_{9 \times 9}
$$

Grâce à cette simplification et après avoir assemblé les vecteurs des forces intérieures et extérieures, on obtient un système d'équations découplées dont la $i^{\text {ième à }}$ résoudre au temps $t_{n}$ est :

$$
U_{i}\left(t_{n+1}\right)=\frac{\Delta t^{2}}{\overline{M_{i i}}}\left(F_{e x t_{i}}-F_{i n t_{i}}\right)+2 U_{i}\left(t_{n}\right)-U_{i}\left(t_{n-1}\right)
$$

où $\overline{M_{i i}}$ est le coefficient de la matrice masse diagonale assemblé, $[\bar{M}]$, correspondant au degré de liberté $i$.

\section{Comportement des matériaux}

Les matériaux concernés par les procédés de thermoformage sont essentiellement les polymères thermoplastiques, dont le plus connu est le polyéthylène. On dit souvent de ces polymères qu'ils sont linéaires, c'est-à-dire qu'il n'y a quasiment pas de liaisons covalentes entre les longues chaînes polymériques, mais seulement des liaisons secondaires. C'est pourquoi les thermoplastiques se ramollissent lors de leur chauffage : les liaisons secondaires liant les chaînes les unes aux autres se brisent et le matériau s'écoule, ce qui permet sa mise en forme. Pour des températures au-dessus de la température de transition vitreuse, le comportement est similaire à celui des caoutchoucs c'est-à-dire élastique ou viscoélastique (avec une faible viscosité) nonlinéaire et incompressible. Les déformations atteintes avant la rupture sont de l'ordre de 300 a $1000 \%$.

\subsection{Modeles hyperélastiques}

Dans un premier temps, les polymères thermoplastiques sont considérés hyperélastiques [SCH 75]. Ce type de matériau est caractérisé par l'existence d'une fonction scalaire énergie de déformation $W$ dépendant de l'état de déformation. Le second tenseur de Piola-Khirchhoff $\overline{\bar{S}}$ s'exprime en fonction de cette énergie, du tenseur des dilatations de Cauchy-Green droit, $\overline{\bar{C}}$, et de la pression hydrostatique $p$ due a l'incompressibilité: 


$$
\overline{\bar{S}}=-p \overline{\bar{C}}^{-1}+2 \frac{\partial W}{\partial \overline{\bar{C}}}
$$

Les deux modèles implantés dans notre code de calcul sont les modèles :

- de Mooney [MOO 40] défini par :

$$
W=C_{1}\left(I_{1}-3\right)+C_{2}\left(I_{2}-3\right)
$$

où $C_{1}$ et $C_{2}$ sont des constantes matérielles et $I_{1}$ le premier invariant de $\overline{\bar{C}}$;

- d’Ogden [OGD 72], utilisant des mesures généralisées de la déformation :

$$
W=\sum_{n=1}^{N} \frac{\mu_{n}}{\alpha_{n}}\left(\lambda_{1}^{\alpha_{n}}+\lambda_{2}^{\alpha_{n}}+\lambda_{3}^{\alpha_{n}}-3\right)
$$

où $N$ est le nombre de termes de la série, les doublets de réels $\left(\mu_{n}, \alpha_{n}\right)_{n=1, N}$ sont les constantes matérielles et $\lambda_{i}, i=1,3$ sont les extensions principales (racines carrées des valeurs propres de $\overline{\bar{C}}$ ).

L'implantation de ces modèles dans le code de calcul se fait simplement. En effet, le calcul du vecteur des efforts intérieurs [8] ne nécessite que le calcul du vecteur des contraintes dans le repère local de l'élément, $\left\{S_{l}\right\}^{t}=\left\langle S_{l_{11}}, S_{l_{22}}, S_{l_{12}}\right\rangle^{t}$. Dans le cas du modèle de Mooney, on calcule directement les trois composantes de ce vecteur en utilisant [17] et [18]. Dans le cas du modèle d'Ogden, comme la fonction énergie de déformation est exprimé en termes d'extensions principales, on se doit de passer par l'intermédiaire de la base propre du tenseur $\overline{\bar{C}}$.

\subsection{Modèles viscoélastiques non-linéaires intégraux}

Dans la réalité, des phénomènes visqueux apparaissent lors de la déformation des polymères fondus à leur température de mise en forme [WAR 83]. L'état de contrainte ne dépend plus seulement de l'état actuel de la structure mais aussi de l'histoire de la transformation qu'a subie le matériau pour atteindre l'état actuel. Il y a donc dissipation d'énergie (lors des ruptures des longues chaînes polymériques par exemple). On peut dire que le matériau possède une forme de «mémoire».

Dans ce travail, nous nous sommes intéressés aux modèles intégraux classiquement utilisés pour la modélisation du comportement des élastomères, généralisation des modèles hyperélastiques précédents :

- modèle de Christensen [CHR 80] :

$$
\overline{\bar{S}}(t)=-p \overline{\bar{C}}^{-1}(t)+g_{0} \overline{\bar{I}}+\frac{1}{2} \int_{0}^{t} g_{1}(t-\tau) \frac{\overline{\bar{C}}(\tau)}{d \tau} d \tau
$$

où $g_{0}$ et $g_{1}(\tau)$ sont les paramètres matériels ; 
- modèle CBT [CHA 76], que nous avons généralisé :

$$
S_{i}(t)=-p \frac{1}{\lambda_{i}^{2}}+\sum_{n=1}^{N} g_{0_{n}} \lambda_{i}^{\alpha_{n}-2}(t)+\sum_{n=1}^{N} g_{0_{n}} \frac{1}{\lambda_{i}^{2}(t)} \int_{0}^{t} g_{1}(t-\tau) \frac{d \lambda_{i}^{\alpha_{n}}(\tau)}{d \tau} d \tau \quad[21]
$$

où $N$ est le nombre de termes de la série, $\left(g_{0_{n}}, \alpha_{n}\right)_{n=1, N}$ et $g_{1}(\tau)$ sont les paramètres matériels.

Pour l'implantation de ces lois de comportement dans le code de calcul, on doit maintenant prendre en compte l'histoire de la déformation du matériau pour calculer les contraintes au temps présent. Feng [FEN 86] propose une relation de récurrence qui permet de calculer la contrainte au temps discret présent $t_{n}$ seulement en fonction des grandeurs (contraintes et déformations) aux temps $t_{n}$ et $t_{n-1}$. Pour cela, on considere que la fonction de relaxation a la forme suivante :

$$
g_{1}(\tau)=g_{1} e^{-t / \tau}
$$

Par exemple, pour le modèle de Christensen, la relation de récurrence, dans les directions principales, s'écrit alors :

$$
\begin{aligned}
S_{i}\left(t_{n}\right)=-p \frac{1}{\lambda_{i}^{2}\left(t_{n}\right)}+g_{0}+ & \frac{1}{2} e^{-\frac{\Delta l}{r_{R}}} \int_{0}^{t_{n}-1} g_{1}\left(t_{n-1}-\tau\right) \frac{d \lambda_{i}^{2}(\tau)}{d \tau} d \tau \\
& +g_{1} e^{-\frac{\Delta l}{\partial \tau_{R}}}\left[\lambda_{i}^{2}\left(t_{n}\right)-\lambda_{i}^{2}\left(t_{n-1}\right)\right] \quad i=1,3
\end{aligned}
$$

La relation est similaire pour le modele CBT généralisé [VER 97].

L'évolution des variables cinématiques lors du pas de temps étant inconnue, nous supposons que les directions principales sont constantes lors de celui-ci et sont égales aux directions principales au temps présent final $t_{n}$. Cette hypothèse a été utiliséc par Rachik [RAC 94] pour des lois de comportement viscoélastiques différentielles.

\section{Gestion du contact}

Pour pouvoir simuler les procédés de mise en forme, il est nécessaire de développer un module de contact qui permettra de prendre en compte la présence du moule.

La plupart des travaux dans ce domaine adopte l'hypothèse de contact collant. En effet, lorsque la paraison à haute température entre en contact avec le moule froid, ses caractéristiques matérielles changent très rapidement et la membrane se raidit. Nous nous sommes placés dans le cadre de cette hypothèse.

La méthode consiste à déterminer l'intersection du segment $\left[N^{\prime} N\right]$ formé par la position d'un nœud aux temps $t_{n-1}$ et $t_{n}$ (trajectoire supposée linéaire) avec les facettes triangulaires $(D E F)$ définissant le moule (figure 3). Cette méthode autorise l'utilisation de facettes grossières pour la discrétisation du moule [VER 97]. 


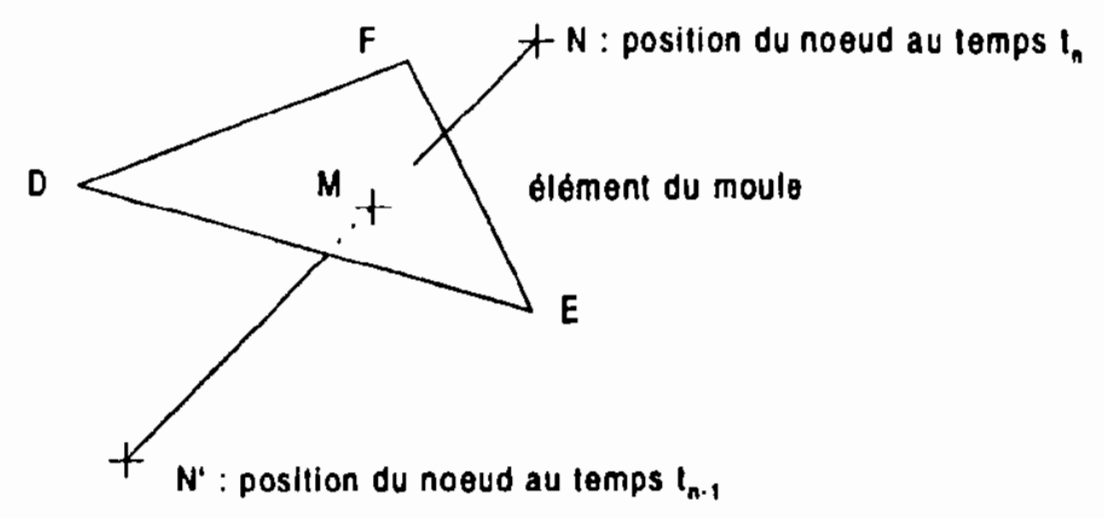

Figure 3. Géométrie du problème de contact

\section{Raffinement du maillage}

Le problème majeur posé par l'utilisation d'ćléments triangulaires linéaires réside dans le fait qu'ils restent plans. En effet, compte tenu des très grandes déformations que ces éléments subissent, ceci ne permet pas de simuler parfaitement les évolutions de la géométric de la paraison, par excmple dans les coins d'un moule (figure 4). Pour remédier à cette difficulté, certains auteurs présentent le raffinement de maillage
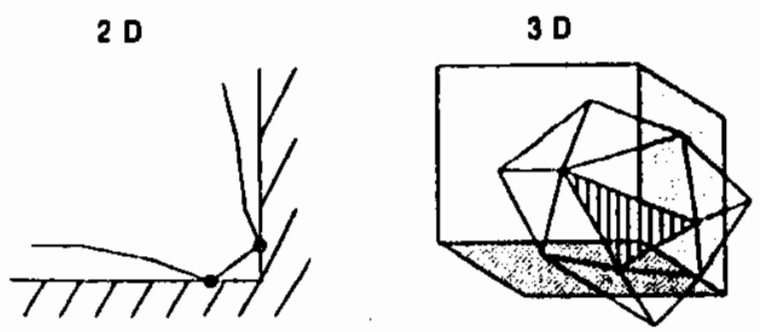

Figure 4. Problème posé par un maillage gmssier dans le coin d'un moule

comme unc perspective de recherche intéressante [DEL 91] sans toutefois le mettre en œuvre.

A partir de ces constatations, nous avons développé un module de raffinement du maillage basé sur un critère géométrique. Lorsqu'on décide de remailler la paraison, chaque élément de la membrane est testé. Pour ce faire, on considère les éléments adjacents à cet élément et on calcule la normale moyenne à ce groupe d'éléments. Si l'angle entre cette normale moyenne et la normale à l'un des éléments du groupe est supérieur à une valeur fixée, alors l'élément correspondant est subdivisé.

Après avoir rempli la liste des triangles qui doivent être subdivisés, nous utilisons l'algorithme développé par Nambiar et al. [NAM 93] pour le raffinement de maillages constitués d'éléments triangulaires à trois nœuds. Leur technique est basée sur une méthode de découpage par bissection suivant le plus grand côté, la méthode de division adoptée differe suivant les trois cas présentés sur la figure 5. Pour mettre en place une telle méthode, il convient de classer les triangles dans l'ordre de leur plus grand côté croissant et de les diviser en partant de la fin de la liste. 


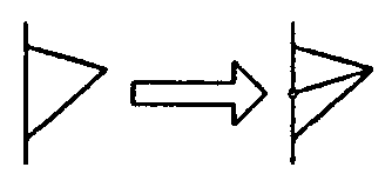

(a)

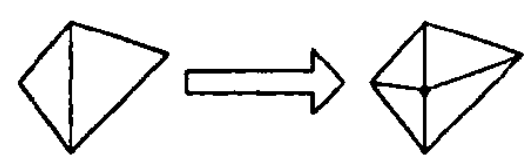

(b)

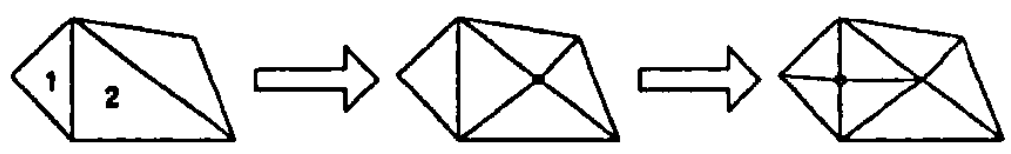

(c)

Figure 5. Méthodes de division des triangles : (a) cas d'un élément sur la frontière, (b) cas de deux éléments internes partageant leur plus grand côté, (c) cas des éléments internes qui ne partagent pas leur plus grand côté

\section{Résultats et discussion}

\subsection{Validation semi-analytique : soufflage dynamique d'une membrane sphérique}

Pour valider notre code, nous nous intéressons dans un premicr temps au problème du soufflage dynamique de membranes sphériques dont on connaît une solution semianalytique.

La mise en équations du soufflage dynamique d'une sphère soumise à un échelon de pression aboutit à une équation différentielle non-linéaire du second ordre d'inconnue $\lambda$, extension principale circonférentielle, rapport du rayon déformé au rayon non déformé [VER 97]. L'équation normalisée, pour le modèle de Mooney, est :

$$
\ddot{\lambda}=\Delta p \lambda^{2}+\left(\frac{1}{\lambda^{5}}-\lambda\right)\left(1+\alpha \lambda^{2}\right)
$$

de même, pour le modèle de Christensen, on obtient :

$$
\begin{aligned}
\ddot{\lambda}=\Delta p \lambda^{2}+\left(\frac{1}{\lambda^{5}}-\lambda\right)-\frac{\lambda}{2} \int_{0}^{t} \alpha(t-\tau) & \frac{d \lambda(\tau)^{2}}{d \tau} d \tau \\
& +\frac{1}{2 \lambda^{5}} \int_{0}^{t} \alpha(t-\tau) \frac{d \lambda(\tau)^{-4}}{d \tau} d \tau
\end{aligned}
$$

Ces équations sont résolues par une méthode de Runge-Kutta d'ordre 5 ou 6 pour différentes valeurs du paramètre matériel $\alpha\left(\alpha=C_{2} / C_{1}\right.$ pour le modèle de Mooney, et $\alpha(\tau)=g_{1} / g_{0} e^{-\tau / \tau_{R}}$ pour celui de Christensen) et du pas de pression normalisé $\Delta p$. Les mêmes calculs sont effectués avec le code de calcul sur une sphère complète de 2000 eléments finis.

Les résultats obtenus sont très satisfaisants : les réponses aux sollicitations sont reproduites avec succès par les éléments finis aussi bien pour un comportement hyperélastique (figure 6) que viscoélastique (figure 7) et ce, quelle que soit la nature de la réponse (oscillatoire ou instable). 


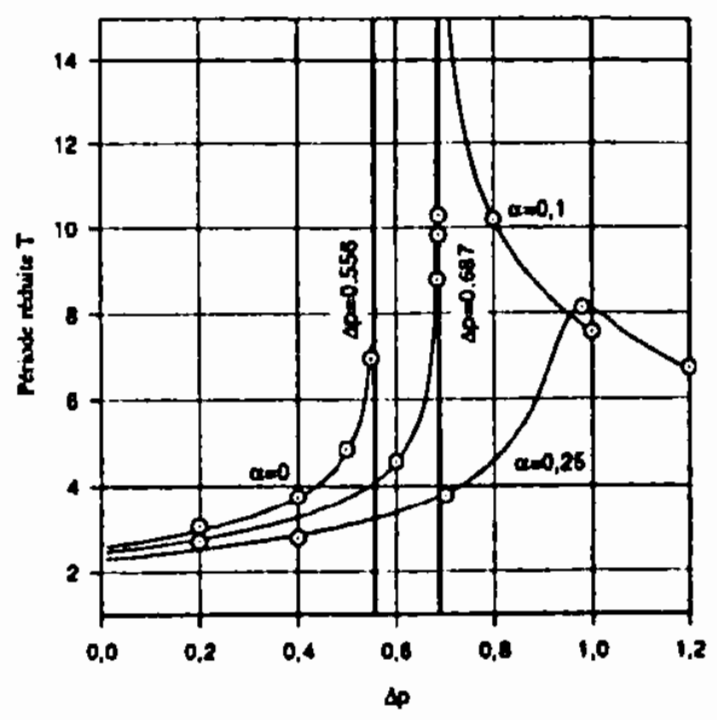

Figure 6. Soufflage d'une membrane sphérique de type Mooney : période réduite en fonction de la pression imposée (-) Runge-Kutta, $\odot$ éléments finis

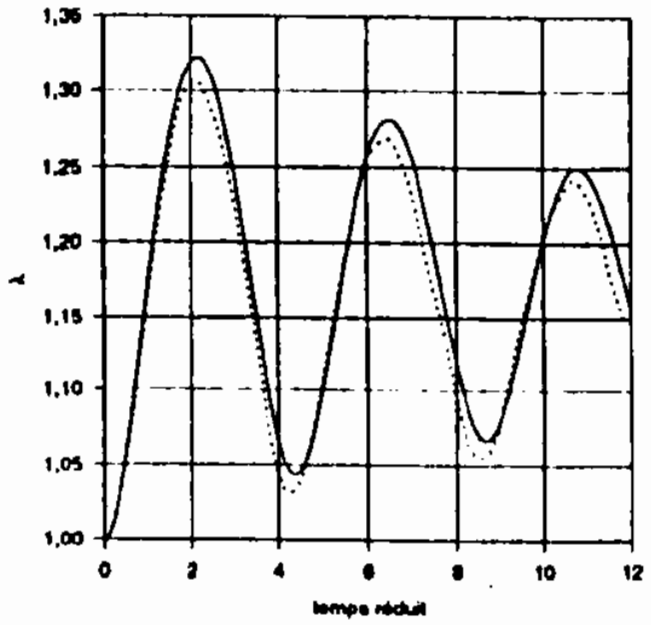

(a) Réponse oscillatoire amortie, $\Delta p=0,5$

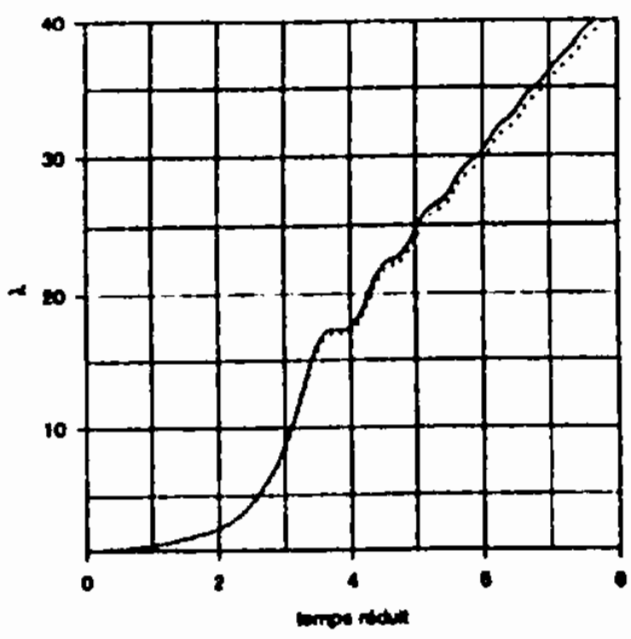

(b) Reponse instable, $\Delta p=1$

Figure 7. Soufflage dynamique d'une membrane sphérique viscoélastique de type Christensen : (-) Runge-Kutta, (...) éléments finis

\subsection{Validation générale : thermoformage d'un récipient cylindrique}

Pour valider l'ensemble du code de calcul, nous avons confronté nos résultats numériques à des données expérimentales fournies dans la littérature. Pour cela, on s'intéresse au cas du thermoformage d'un récipient cylindrique en HIPS (PolyStyrène à Haut Impact). Ce problème a été étudié expérimentalement et numériquement par deLorenzi et Nied [DEL 91] et numériquement par Rachik et al. [RAC 94].

Pour des raisons de symétrie, nous n'avons étudié que le quart de la structure. La membrane initiale est plane, circulaire de rayon 129,54 mm et d'épaisseur initiale 
$0,254 \mathrm{~mm}$. La géométrie du moule est présentée sur la figure 8(a). Celui-ci est maillé avec 63 facettes triangulaires (figure $8(\mathrm{~b})$ ).

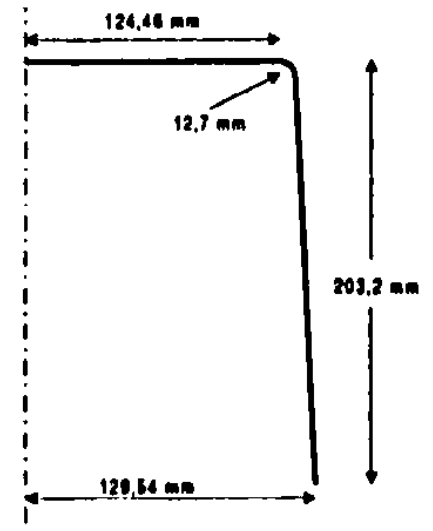

(a) Géométrie du moule

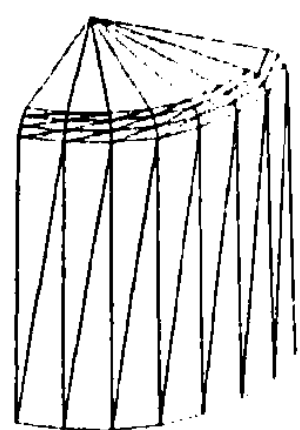

(b) Maillage du moule

Figure 8. Description du moule pour le thermoformage d'un récipient cylindrique

Pour modéliser le comportement du matériau, nous avons utilisé les données expérimentales fournies par Schmidt et Carley [SCH 75] qui proposent une forme modifiée du modèle de Mooney, avec la fonction énergie de déformation suivante :

$$
W=A_{10}\left(I_{1}-3\right)+A_{02}\left(I_{2}-3\right)^{2}
$$

où les deux constantes matérielles sont :

$$
A_{10}=0,143 \mathrm{MPa} \text { et } A_{02}=2,210^{-6} \mathrm{MPa}
$$

Cette loi de comportement et ces constantes matérielles sont celles utilisées par les autres auteurs dans leurs études.

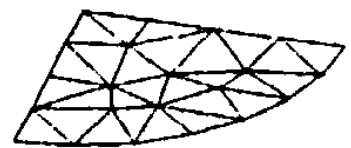

(a) 27 éléments

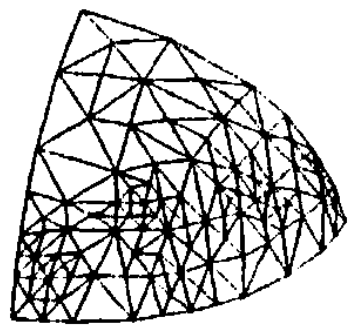

(b) 201 éléments

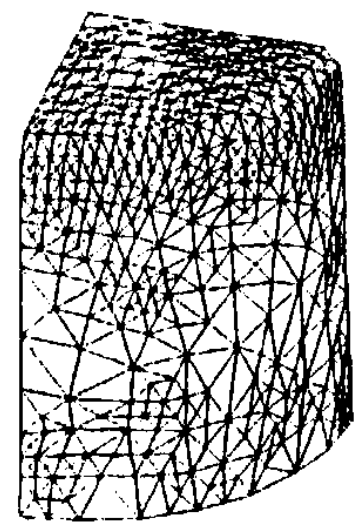

(c) 996 éléments

Figure 9. Trois phases du thermoformage 
Le maillage initial de la membrane est constitué de 27 ćléments. La figure 9 montre l'évolution du maillage au cours du soufflage. On remarquera la qualité du maillage final (figure 9(c)). Il convient de noter qu'il n'était pas possible de prévoir $a$ priori les zones de la paraison initiale (figure 9(a)) pour lesquelles le maillage devait être fin.

Nied et deLorenzi ont mesuré expérimentalement la répartition de l'épaisseur le long de la paroi de la pièce finale, ainsi que les extensions principales radiale et circonférentielle au fond du récipient.

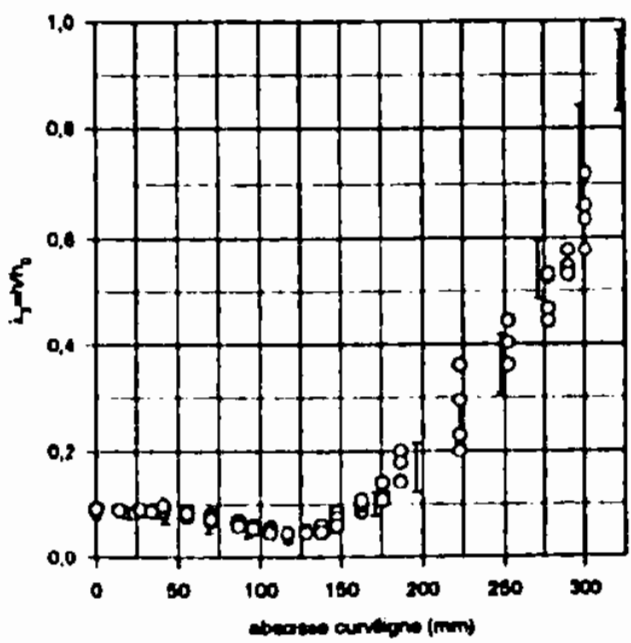

Figure 10. Epaisseur réduite le long de la parmi : (I) points expérimentaux, (o) résultat numérique $E F$

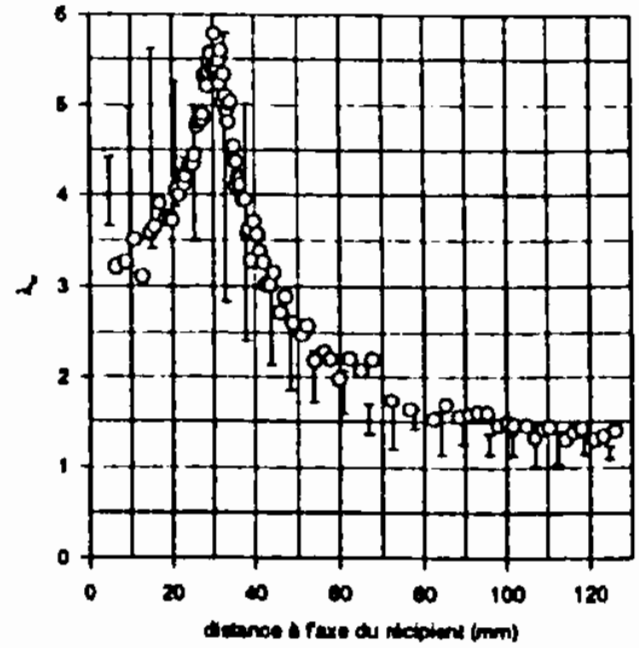

(a) Extension principale radiale au fond du reccipient

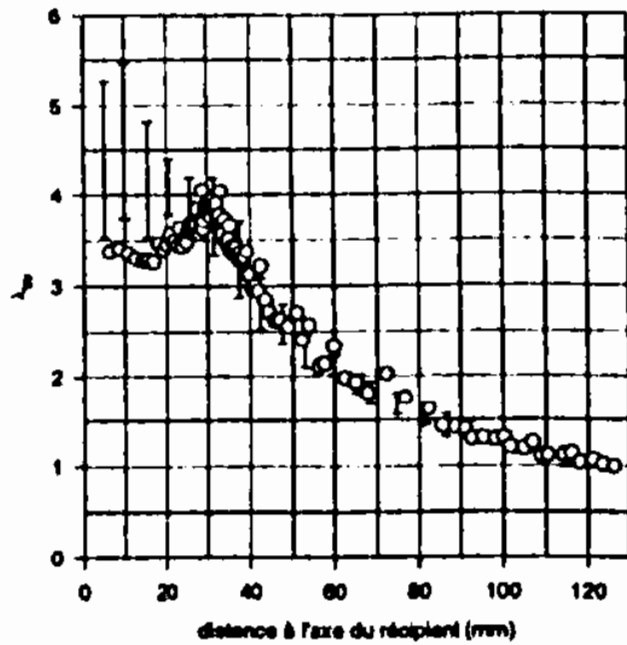

(b) Extension principale circonférentielle au fond du récipient

Figure 11. Comparaison expériences-calculs : (I) points expérimentaux, (०) résultats numériques $E F$

La répartition de l'épaisseur obtenue numériquement est en bonne adéquation avec les résultats expérimentaux (figure 10), et ce, surtout pour le fond du récipient. Pour 
des abscisses curvilignes supérieures à $250 \mathrm{~mm}$, les résultats numériques s'éloignent des mesures expérimentales ( $15 \%$ ). Cet écart croissant peut être imputé à l'aspect grossier du maillage final sur le bord du récipient.

En ce qui concerne les extensions principales au fond du récipient (figure 11), les résultats s'avèrent un peu moins satisfaisants : l'extension principale radiale est quelque peu sous-estimée sur la plus grande partie du fond $(17 \%)$ et surestimée au voisinage de l'axe de symétrie (25\%) (figure 11 (a)), et l'extension principale circonférentielle est sous-estimée au voisinage de l'axe (25\%) (figure 11(b)). Malgré ces écarts au voisinage de l'axe de symétrie, sur un plan qualitatif, les résultats numériques sont conformes aux résultats expérimentaux.

Au vu de cet exemple industricl, notre code de calcul utilisé avec son module de raffinement de maillage est validé.

\subsection{Thermoformage d'une boîte avec insert}

Le second exemple est consacré au thermoformage d'une boîte avec un insert au fond. Les dimensions du moule sont présentées sur la figure 12(a). Ce moule est maillé à l'aide de 26 facettes triangulaires (figure 12(b)). La paraison initiale cst une

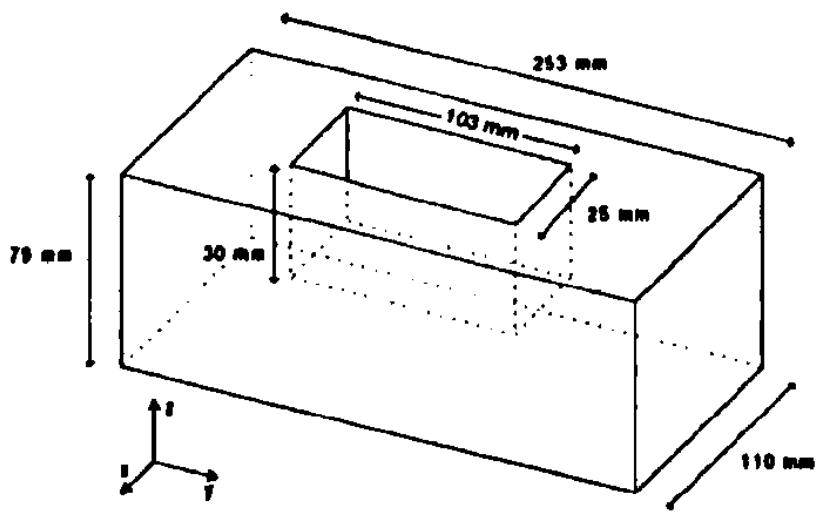

(a) Géométrie du moule

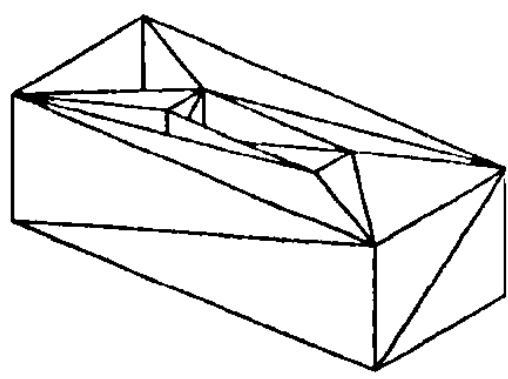

(b) Maillage du moule

Figure 12. Description du moule pour le thermoformage d'une boite parallélépipèdique avec un insert

feuille rectangulaire d'épaisseur uniforme $2 \mathrm{~mm}$, dont les bords sont fixés. Pour ce calcul, le chargement imposé consiste en une rampe de pression.

La figure 13 présente l'évolution du maillage au cours du thermoformage ; le maillage initial comprend 128 éléments (figure 13(a)) et le maillage final est constitué de 3752 éléments (figure 13(c)).

La figure 14 fournit la répartition de l'épaisseur sur les demi-plans de symétrie $(x z)$ (figure 14(a)) et (yz) (figure 14(b)), plans identifiés par le repère de la figure 12(a). 
Cet exemple incluant un moule non-convexe démontre la fiabilité du module de contact du code de calcul. De plus, l'utilisation du module de raffinement de maillage permet d'obtenir une géométrie finale satisfaisante sans avoir à se soucier du maillage initial.

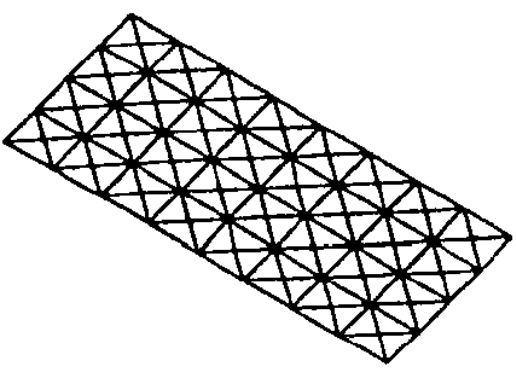

(a) 128 éléments

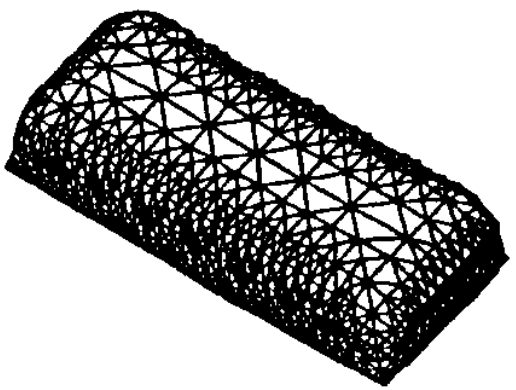

(b) 1772 élếments

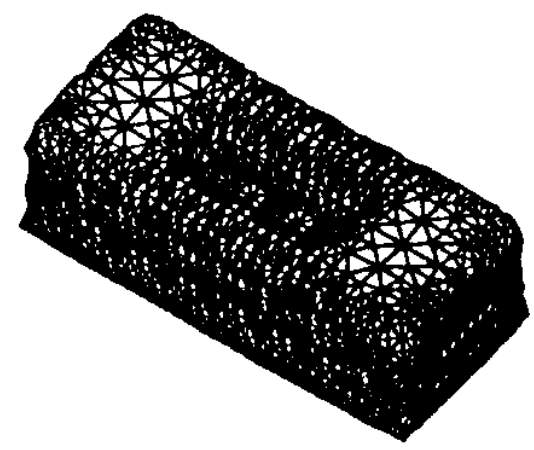

(c) 3752 éléments

Figure 13. Trois phases du thermoformage de la boîte

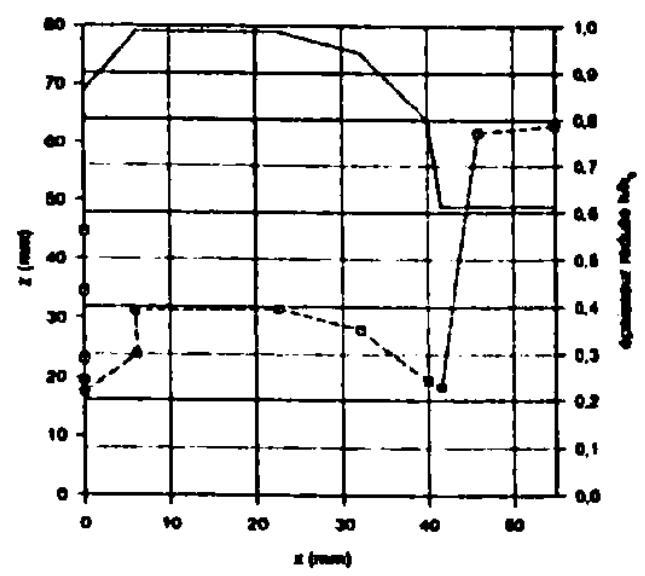

(a) Plan (xz)

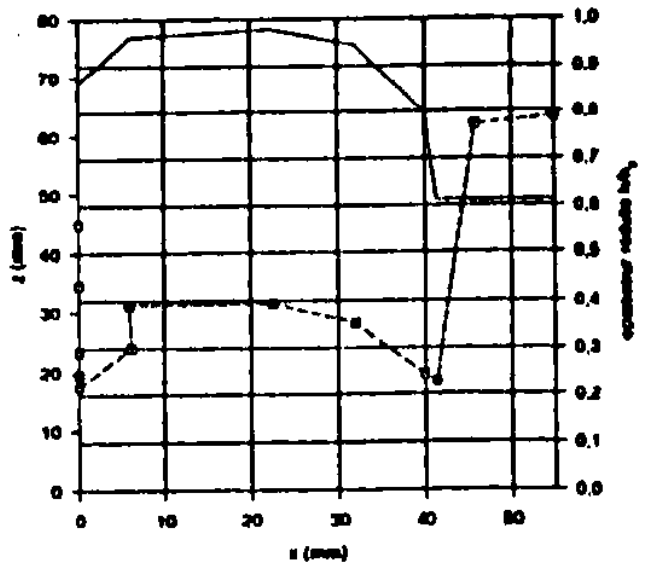

(b) Plan (yz)

Figure 14. Répartition de l'épaisseur réduite sur les demi-plans de symétrie : (一) coupe de la boîte. (耳) épaisseur

\section{Conclusion}

Le code de calcul présenté permet de simuler numériquement le procédé de thermoformage et de connaître avec précision l'évolution de la géométrie du produit au cours de la mise en forme, notamment grâce à la technique de remaillage employée. Ce programme peut également être utilisé pour la simulation du procédé de soufflage permettant l'obtention de bouteilles. 


\section{Bibliographie}

[CHA 76] Chang W. V., Bloch R., Tschoegl N. W., "On the theory of the viscoelastic behavior of soft polymers in moderately large deformations $»$, Rheol. Acta, 15, 1976, p. 367-378.

[CHR 80] CHRISTENSEN R. M., «A nonlinear theory of viscoelasticity for application to elastomers », J. Appl. Mech. ASME, 47, 1980, p. 762-768.

[DEL 91] Delorenzi H. G., NiED H. F., « Finite element simulation of thermoforming and blow molding », Progress in Polymer Processing. Hanser Verlag, (1991), p. 117-171.

[DOK 89] DoKaINISH M. A., SUbBARAJ K., « A survey of direct time-integration methods in computational structural dynamics. I. Explicit methods», Comput. Struct., 32, 1989, p. 1371-1386.

[FEN 86] FENG W. W., "A recurrence formula for viscoelastic constitutive equations ", Proceedings of the International Conference on Computational Mechanics vol. IV. (1986), p. 77-81.

[KHA 92] Khayat R. E., Derdouri A., GARCia-Réjon A., «Inflation of an clastic cylindrical membrane: non-linear deformation and instability », Int. J. Solids Structures, 29, 1992, p. 69-87.

[MOO 40] MOONEy M., "A theory of large elastic deformation », J. Appl. Phys., II, 1940, p. $582-592$.

[NAM 93] Nambiar R. V., Valera R. S., LAWrence K. L., Morgan R. B., Amil. D., "An algorithm for adaptative refinement of triangular element meshes ». Int. J. Num. Meth. Eng., 36, 1993, p. 499-50\%.

[OGD 72] OGDEN R. W., « Large deformation isotropic elasticity - on the correlation of theory and experiment for incompressible rubberlike solids », Proc. R. Soc. Lond., A326, 1972, p. 565-584.

[RAC 94] RaChIK M., RoelandT J. M., Batoz J. L., "Simulation numérique du soufflage et du thermoformage des plastiques $»$, Revue européenne des éléments finis, 3, 1994, p. 187-210.

[SCH 75] SCHMIDT L. R., CARLEY J. F., «Biaxial stretching of heat-softened plastic shects: experiments and results », Polym. Eng. Sci., 15, 1975, p. 51-62.

[VER 97] VERRON E., «Contribution expérimentale et numérique aux procédés de moulage par soufflage et de thermoformage ». Thèse de doctorat, École Centralc de Nantes, 1997.

[WAR 83] WARD I. M., Mechanical properties of solid polymers, sccond edition, John Wiley and Sons Ltd, 1983.

[ZaM 89] Zamani N. G., Watt D. F., Esteghamatian M., * Status of the finite element method in the thermoforming process ». Int. J. Num. Meth. Eng., 28, 1989, p. 2681-2693.

[ZIE 94] ZIENKIEWIECZ O. C., TAYLOR R. L., The finite element method. Volume I: Basic formulation and linear problems, fourth edition, MacGraw-Hill Company, 1994. 